\title{
Cognitive activity and physiological arousal: Processes that mediate mood-congruent memory
}

\author{
LARRY J. VARNER and HENRY C. ELLIS \\ University of New Mexico, Albuquerque, New Mexico
}

\begin{abstract}
This research proposes that the cognitive activity associated with the experience of an emotional state mediates the occurrence of mood-congruent processing. Two experiments examined the role of cognitive activity in selective processing of words in a mood congruence paradigm. Four induction procedures were used: a depressed-mood induction, a schema induction organized around the theme of writing a paper, an arousal induction, and a control neutral-mood induction. The memory task consisted of recalling a word list composed of negatively associated and thematically organized words. Selective processing was demonstrated in conjunction with the depressed-mood and organizational-schema induction procedures. In contrast, the arousal and neutral induction procedures did not produce selective processing of words from the list. The findings support the thesis that cognitive activity mediates the selective processing typical of mood congruence as distinct from arousal processes per se. The findings are discussed with respect to the resource allocation model and semantic network theory.
\end{abstract}

We are interested in understanding what processes mediate the phienomenon of mood-congruent memory. Moodcongruent memory is a well-established effect in mood and memory research (Bower, Gilligan, \& Monteiro, 1981; Bower \& Mayer, 1989, Experiments 1 \& 3; D. M. Clark \& Teasdale, 1982; Fiedler \& Stroehm, 1986; Gilligan \& Bower, 1983; Martin, Horder, \& Jones, 1992; Mayer \& Volanth, 1985; Parrott, 1991; Perrig \& Perrig, 1988; Rothkopf \& Blaney, 1991; Salovey \& Singer, 1989). Mood congruence refers to the selective processing of information that is affectively consistent with one's current mood state at the expense of information that is not related to one's current mood (Blaney, 1986). Although mood congruence is a reliable phenomenon, the processes that mediate mood congruence are still unclear, and at least two alternate explanations are possible that are derived from the current multidimensional conceptualization of emotional states. An emotional state is regarded as consisting of a

The research was supported in part by a grant from the Student Resource Assistance Committee at the University of New Mexico to the first author and a grant from the College of Arts and Sciences to the second author. Portions of the research were presented by $\mathrm{H}$. C. Ellis as part of a Science Directorate invited address at the 103rd Annual Convention of the American Psychological Association, New York, August 1995, and at an invited symposium on memory (Alice Healy, Chair) at the Rocky Mountain Psychological Association meeting in Boulder, CO, May 1995. Portions of this research were also presented by L. J. Varner at the Southwestern Cognition Society, Arlington, TX, May 1993. The authors wish to thank Gordon Bower, Eric Eich, and Tom Nelson, who reviewed an earlier version of this manuscript. Correspondence should be addressed to L. J. Varner, Department of Psychology, Logan Hall, University of New Mexico, Albuquerque, NM 87131 (e-mail: mandor@sprynet.com), or to H. C. Ellis, Department of Psychology, Logan Hall. University of New Mexico, Albuquerque. NM 87131 (e-mail: hellis( $)$ unm.edu).

—Accepted by previous editor, Geoffrey R. Loftus change in physiological arousal state and an activation of associated cognitive processes (see Mandler, 1992; Mayer \& Salovey, 1988; Schacter \& Singer, 1962). Either of these two components could underlie mood congruence (see Figure 1)

The first possibility is that mood congruence is directly produced by changes in arousal associated with the presence of an emotional state, either a prevailing mood or one produced by experimental procedures. Conceptualizing arousal as the process underlying mood congruence is consistent with general theories that emphasize the arousal aspect of emotional states (e.g., Zajonc, 1980).

If physiological arousal mediates mood congruence, then there are several predicted effects of physiological arousal with respect to the selective processing of information. First, there should be a selective processing of affective information over nonaffective information. Affective information involves an arousal component that would be congruent with the experience of arousal, whereas nonaffective information does not. Second, the selective processing of information instigated by the experience of physiological arousal would be different from that of individuals not experiencing a change in physiological arousal. Finally, the effects on the processing of information would be separate from those associated with cognitions produced by an emotional experience.

The second possibility is that mood congruence is a result of cognitive processes activated as a consequence of the emotional state experienced and not physiological arousal. This is the dominant view and is reflected in research examining emotion and information processing. For example, Bower (1981) proposed a network model that included moods as nodes contained in a semantic network, emphasizing their cognitive content; similarly, Riskind (1989) proposed that cognitions primed by the emotional experience serve as cues for selective processing of mood- 


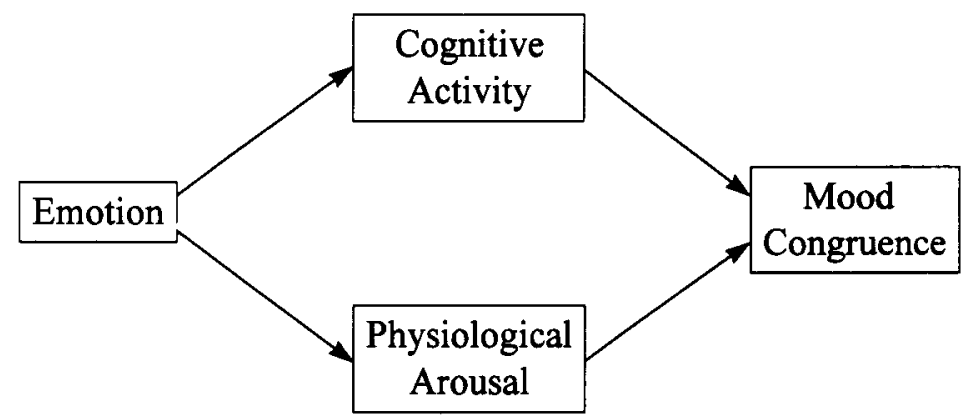

Figure 1. Configuration of the possible steps resulting in mood congruence. An emotional state leads to both cognitive activity and physiological arousal, and either or both could lead to the occurrence of mood congruence. Current dominant theories state that emotional states are composed primarily of cognitive activity and physiological arousal. Previous research has shown that mood congruence is a reliable phenomenon. The present research focuses on examining the relative contributions of the primary aspects of an emotional state in producing mood congruence.

congruent information. Although researchers have discussed the role of cognitive activity associated with an emotional experience, Ellis and colleagues (Ellis \& Ashbrook, 1988; Ellis, Varner, \& Becker, 1993) have proposed that emotional states produce their effects by way of cognitive activities that are a result of the emotional experience. It is expected, therefore, that mood congruence would be mediated by cognitive processes initiated during an emotional state, whereas physiological arousal would have little or no impact on its occurrence.

To show that cognitive activity plays a central role in mood congruence, several specific results must be demonstrated. First, conditions that lead to an activation of cognitive processes related to a specific concept must result in the selective recall of information related to the concept above that of unrelated information. Second, the pattern of selective recall by individuals experiencing the activation of cognitive processes must be different from the pattern of selective recall by individuals who did not experience organized activation of cognitive processes. Finally, it must be demonstrated that the pattern of recall associated with physiological arousal is different from that associated with cognitive activity and from a pattern of recall necessary to demonstrate selective recall.

The assumption that cognitive activity instigated by of an emotional state is responsible for the selective processing of information associated with mood congruence was examined by using a number of induction procedures that lead to changes in cognitive activity and/or physiological arousal. First, in order to replicate mood congruence and to have a pattern of selective processing against which to compare the results of the other conditions, a depressed mood was used. Obviously, we must first demonstrate a mood congruence effect in order to examine the processes that mediate it. A second condition involved the production of organized cognitive activity related to one particular topic: writing a paper. This condition was designed to encourage cognitive activity in the absence of a change in physiological arousal. A third condition involved a change in physiological arousal produced through physical activity. Any thoughts produced by the physical activity will presumably be idiosyncratic and unrelated to any information that will be processed as part of the experiment. Finally, a neutral condition was used as a baseline control. The neutral condition was designed to involve no changes in physiological arousal. Moreover, any thoughts produced are also most likely be idiosyncratic and unrelated to task materials used. Two tests were used to compare the relative effectiveness of the different inductions used: a self-evaluative level of negative mood and a measure of heart rate (HR). The depressed-mood condition was expected to lead to an elevated score on the self-report measure, and the physical activity was expected to lead to an elevated score on the measure of HR. Both measures have been used in previous research in similar situations (see Clark, Milberg, \& Erber, 1988; Clark, Milberg, \& Ross, 1983; Ellis, Seibert, \& Varner, 1995).

To test for mood congruence and selective processing, a word list was used in which half the list of words was related to the negative mood and half was composed of words related to information about writing a paper. The word list used was similar to one used by Bower and Mayer (1989, Experiments 1 and 3) in their replication of moodcongruent memory in terms of selection and presentation of the words.

In summary, the overall expectation was that only the conditions involving cognitive activity would result in selective processing of information, whereas conditions that did not involve cognitive activity would fail to produce selective processing. Specifically, the depressed-mood condition was expected to lead to a greater processing of negative information than organizational information. the condition involving thoughts related to writing a paper was expected to lead to greater processing of organizational information than negative information, and neither the arousal condition nor the neutral condition was 
expected to show a bias to process a particular type of information. If obtained, these findings would emphasize the importance of cognitive activity in the selective processing typical of mood congruence and would de-emphasize the role of physiological arousal.

\section{EXPERIMENT 1}

Experiment 1 was designed to examine the two possible processes that may underlie the mood congruence phenomenon. This was accomplished by administering one of four induction procedures prior to learning a list of words containing both negatively toned and organizational words. After learning the list, a period of time for recalling the list was given. The expectation was that participants who received the depressed-mood induction procedure would recall more negative than organizational words, whereas those who read about writing a paper would recall more organizational than negative words. In addition, it was expected that participants in the neutral or arousal conditions would exhibit a pattern of recall demonstrating little or no selective bias. The pattern of findings expected would be consistent with the proposal that cognitive activity, and not changes in physiological arousal, mediates the occurrence of mood congruence in memory.

\section{Method}

\section{Participants and Design}

Forty undergraduate students enrolled in introductory psychology classes at the University of New Mexico participated in this experiment. The students were randomly assigned to one of four conditions that differed with respect to the induction procedure used: depressed mood, organizational schema, arousal, and neutral mood Six additional participants were excluded from participation in the experiment on the basis of the prescreening procedure to be described later.

\section{Assessment and Induction Procedures}

Following a prescreening, the depressed-mood, neutral-mood, and organizational-schema induction procedures were presented on an IBM PS/2 computer, allowing for standardization of presentation. The arousal induction procedure did not involve computer presentation, but it involved physical exercise that was performed in the same room in which the other induction procedures were administered

Prescreening. The Beck Depression Inventory (BDI; Beck, 1978) was used to prescreen all potential participants. The BDI provided a measure of the participant's self-evaluative level of depression at the time of the experiment by having the participant select the most appropriate statement from a group of four related statements. Each group of statements reflected a particular idea, and the statements ranged from neutral to relatively depressed. The ratings of the statements within each group ranged from 0 to 3,0 being associated with the neutral statement in the group. A sample set of statements is, with the respective rating in parentheses: (0) I do not feel sad, (1) I feel sad, (2) I am sad all the time and I can't snap out of it. and (3) I am so sad that I can't stand it. Upon completion of the BDI, all numbers selected were summed to provide a single measure of emotional state. Only the participants scoring 12 or less were accepted for participation; this minimized the likelihood of giving a depressedmood induction procedure to someone who was already depressed. A cutting point of 12 or below on the BDI was used because this level has been used as a suitable level in our previous research (e.g., Ellis, Varner, Becker, \& Ottaway, 1995).

Mood induction. After completion of the BDI, half of the individuals who participated in the experiment took part in a mood induction procedure, either depressed or neutral, developed by Seibert and Ellis (1991). The induction procedure consisted of 25 statements read aloud by the participants, those in the depressed-mood induction procedure being self-referential, presented one at a time on a computer screen. An example of a depressed statement was, "I feel a little down today," and an example of a neutral statement was, "Ladybugs are good for the garden."

Before reading the statements, each individual read a set of instructions designed to prepare them to participate fully. The participants were then paced through the statements at a rate of one every $20 \mathrm{sec}$. The neutral-mood induction was used as a baseline for the effects of the other induction procedures. Although it is impossible for the neutral-mood induction procedure to produce no cognitive activity, any cognitions produced by the neutral-mood induction procedure are likely to have an idiosyncratic organization.

Schema induction. The organizational-schema induction procedure was developed by instructing a class of 30 students in an introductory psychology laboratory to write as many statements as they could related to the topic of writing a paper and to try to word each statement possessively (i.e., using "I" as the subject of the sentence). The statements were then compiled according to the frequency of occurrence. The 25 most common statements were selected except those that included a reference to an emotional state (e.g., "I get depressed when I have to write a paper" was eliminated). The 25 accepted statements were randomized and assembled in a manner consistent with the neutral-and depressed-mood statements, and a comparable set of instructions were developed, all of which can be seen in Appendix A. The participants were paced through the statements at a rate of one every $20 \mathrm{sec}$. Accordingly, the schema induction procedure was comparable to the depressed-and neutral-mood induction procedures with regard to setup and administration. The important feature of the schema induction was that it was designed to produce cognitive activity related to writing a paper while correspondingly not influencing the level of arousal.

Arousal induction. The arousal induction consisted of an exercise task previously implemented by M. S. Clark et al. (1988) and M. S. Clark et al. (1983) with successful results. The exercise consisted of stepping up and down on a wooden platform (6 in. high) for $5 \mathrm{~min}$. This induction procedure was used to produce a relatively aroused physiological state without necessarily producing organized cognitive activity. General arousal produced by exercise was used because it has been found that different levels of arousal associated with different sources often produce similar effects with respect to encoding and retrieval (see Revelle \& Loftus, 1990, 1992).

Assessment. Two separate measures were used to assess the participants' mood and arousal levels. The Depression Adjective Checklist (DACL; Lubin, 1965) was used to measure the participants' mood. The DACL consisted of 21 negative and 11 positive adjectives, and the participants were instructed to check the words that best described their current mood. Scores on the DACL were obtained by summing the number of negative words checked and the number of positive words not checked, higher scores reflecting a higher level of depression.

To assess level of arousal, all participants measured their HR prior to and immediately following their assigned induction procedure and after the memory task. The participants were shown how to find their pulse on their carotid artery.

\section{Word List}

The task required the participants to attempt to remember a list of words presented twice, in two different orders, followed by a free recall period. The word list used consisted of 10 negative words (associated with being depressed) and 10 writing-organization words 
Table 1

Mean Heart Rate $(M)$ and Standard Error of the Mean (SEM) as a Function of 'Time of Measure for Experiment 1

\begin{tabular}{|c|c|c|c|c|c|c|}
\hline \multirow{3}{*}{$\begin{array}{l}\text { Induction } \\
\text { Condition }\end{array}$} & \multicolumn{6}{|c|}{ Time of Heart Rate Measure } \\
\hline & \multicolumn{2}{|c|}{ Preinduction } & \multicolumn{2}{|c|}{ Postinduction } & \multicolumn{2}{|c|}{ Posttask } \\
\hline & $M$ & $S E M$ & $M$ & $S E M$ & $M$ & $S E M$ \\
\hline Depressed & 68.3 & 2.2 & 71.5 & 3.4 & 68.2 & 2.4 \\
\hline Schema & 73.1 & 3.1 & 72.4 & 2.9 & 74.2 & 2.8 \\
\hline Arousal & 70.7 & 2.2 & 98.8 & 6.3 & 83.2 & 4.8 \\
\hline Neutral & 74.8 & 3.5 & 74.7 & 4.1 & 71.3 & 4.5 \\
\hline
\end{tabular}

(associated with writing a paper), which had a scaled concreteness rating of less than 3 on a 7-point scale as described by Paivio, Yuille, and Madigan (1968) and can be seen in Appendix B. Bower and Mayer (1989, Experiments 1 and 3 ) demonstrated mood congruence utilizing the same criteria for selecting the words, but they used a shorter list consisting of 8 negative and 8 neutral words. We used a 20 -item list because our participants were required to learn only one list, whereas Bower and Mayer required participants to learn two separate lists.

To control for possible order effects, the order of words was randomized immediately before their presentation to the participants by a computer program. The words were then presented at the center of the computer screen at a rate of one word every $2 \mathrm{sec}$.

\section{Procedure}

After the participants were seated in front of the computer, each was told of the induction procedure to follow and given the option of ending the experiment at any time. The BDI was then completed by all participants, and their initial HR was measured and recorded. Following the administration of the appropriate induction procedure, the participant's postinduction HR was taken, and the DACL was administered. All participants then read instructions describing the upcoming memory task. The word list was then presented twice, in two different orders; after the second presentation, the participants were given up to $3 \mathrm{~min}$ to write as many words from the list as they could. Subsequent to the recall period, all participants who received a depressed-mood induction were given 10 cards with happy statements on them to read. This was done to ensure that no participant left the experiment while still depressed. Finally, all participants had their HR checked and were allowed to leave when it approached their initial HR.

\section{Results and Discussion}

A significance level of .05 was adopted throughout the present and subsequent experiment, with appropriate adjustments when follow-up tests were performed.

\section{Comparability of Groups}

The BDI was used to assess the initial comparability across the four groups. The average scores for the depressed, schema, arousal, and neutral groups were 6.0, $4.5,3.5$, and 4.4 , respectively. There was no reliable difference among the groups $[F(3,36)<1.0]$. On the basis of this screening procedure, the groups can be considered comparable in mood prior to the induction procedures.

\section{Mood Assessment}

The mood produced by the different induction procedures was measured by the DACL. The average scores on the DACL for the depressed, schema, arousal, and neutral inductions were $11.5,6.2,6.6$, and 7.1 , respectively, with the difference among the four groups being reliable $\left[F(3,36)=4.03, M S_{\mathrm{e}}=15.04\right]$. Our expectation was that a reliable mood effect would be due solely to higher scores on the DACL for the participants in the depressed-mood induction; therefore, three planned pairwise comparisons were conducted. The mean DACL score for the neutral group was compared with each of the other three groups, individually. A comparison of the depressed group's score with the neutral group's score yielded a reliable difference $\left[F(1,36)=6.44, M S_{\mathrm{e}}=15.04\right]$. In contrast, neither the schema group nor the arousal group differed reliably from the control group on the DACL measure $(F<1.0)$. In summary, (1) the depressed-mood induction procedure was successful at producing an elevated level of depression, and (2) there was no reliable difference in the measured level of depression among the remaining groups.

\section{Heart Rate Assessment}

HR was a second measure of the effect of the different induction procedures. The left two columns of Table 1 show the HR of the four groups prior to and following the induction procedures. As expected, an examination of Table 1 reveals that, prior to the induction procedures, there was no reliable difference in $\mathrm{HR}$ across groups $[F(3,36)<$ 1.0]. Following the inductions, only the arousal group showed an increased HR, whereas the remaining groups showed little change. The large increase in HR for the arousal group from preinduction to postinduction was reliable $\left[F(1,36)=12.70, M S_{\mathrm{e}}=76.08\right]$. There was also an overall reliable difference in HR across groups $[F(3,36)=$ $\left.131.58, M S_{\mathrm{e}}=76.08\right]$, and there was a reliable interaction of group $\times$ time of measure $\left[F(3,36)=4.80, M S_{e}=76.08\right]$.

Our expectation was that an increase in HR would occur in the arousal group from preinduction to postinduction and that the remaining three groups would show no such increase in HR. We therefore performed three planned pairwise comparisons in order to test our expectation. The analyses were that of comparing the preinduction and postinduction HR for the neutral group with the preinduction and postinduction HR for each of the other three groups. As expected, we found a reliable interaction of induction condition $\times$ time of measure when the neutral group was compared with the arousal group $\left[F(1,36)=28.79, M S_{\mathrm{e}}=76.08\right]$. Also, there was no reliable change in HR over time when the neutral group was compared with the depressed or organizational-schema group $(F<1.0)$. These findings indicate that the arousal induction procedure was successful at producing an elevation in the participant's HR as measured immediately after the induction procedure, whereas the remaining conditions did not.

In addition to measuring HR immediately after the induction procedure, $\mathrm{HR}$ was measured after the completion of the free recall task. As can be seen in the third column of Table 1, the HR of the participants in the arousal con- 


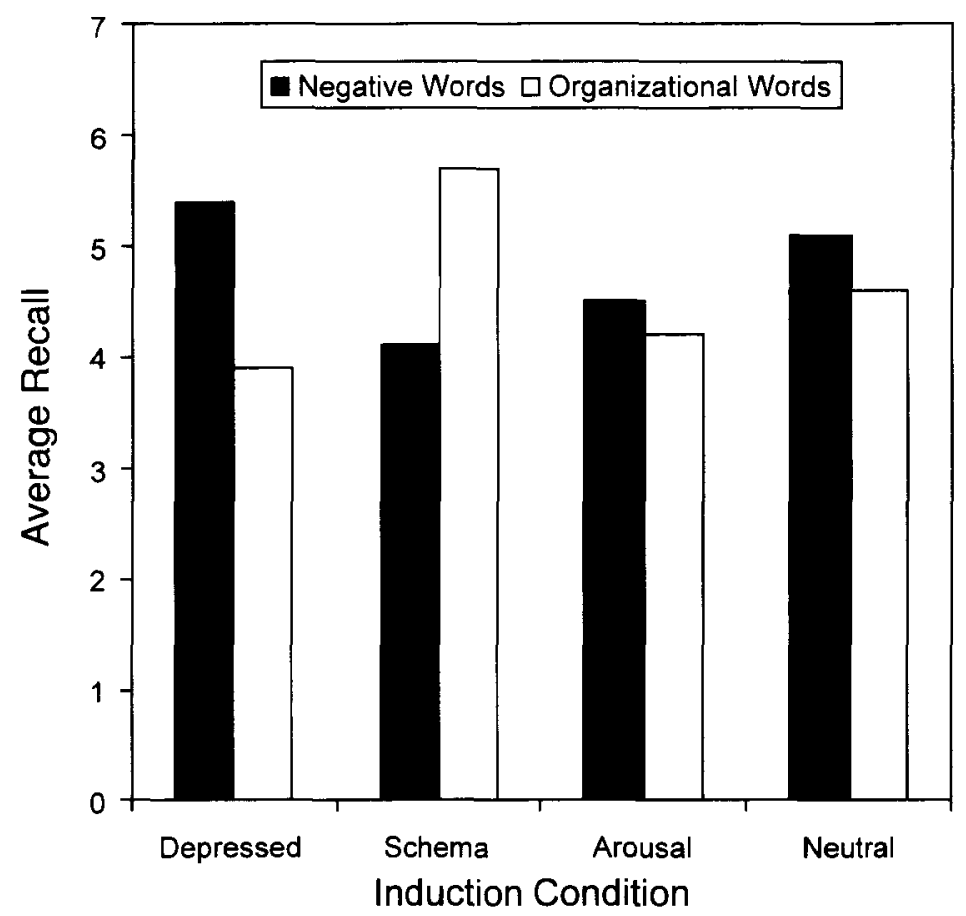

Figure 2. Mean recall for the four induction conditions broken down by word type when examining mood-congruent encoding.

dition continued to be higher than those in the remaining three conditions after the free recall period. When the participants' final HR, measured after the free recall task, was compared with their initial HR across the four conditions, a reliable condition $\times$ time of HR measure interaction was obtained $\left[F(3,36)=5.05, M S_{\mathrm{e}}=47.79\right]$, indicating that the HR change from the initial to final measure was different across the conditions. Although there was a reliable interaction, neither the time of $\mathrm{HR}$ measure nor the condition separately influenced $\operatorname{HR}[F(1,36)=2.62$, and $F(3,36)=1.31$, respectively]

Consistent with the analyses examining the participants' postinduction HR, three follow-up tests comparing the HR of the experimental conditions individually to the HR of the neutral condition were performed. Only the test comparing the HR of the arousal and neutral conditions was reliable $\left[F(1,36)=13.39, M S_{\mathrm{e}}=47.79\right]$; neither the HR in the depressed condition nor the HR in the schema condition differed reliably from the HR measured in the neutral condition across time $(F<1.0)$. The importance of the postinduction and final HR findings is that they indicate that the arousal induction procedure was successful at elevating $\mathrm{HR}$ and that the change in $\mathrm{HR}$ lasted through the free recall task to the end of the experiment.

\section{Free Recall}

The result of primary interest was the free recall of the word list for the four conditions. Figure 2 shows the recall for each group broken down by word type. It is obvious from examining Figure 2 that the different induc- tion procedures had distinct effects on the recall of the two word types, indicating a reliable interaction of word type $\times$ induction condition $\left[F(3,36)=9.93, M S_{\mathrm{e}}=1.10\right]$. Although the interaction was reliable, there was no reliable difference in the recall of the two word types $[F(1,36)=$ 1.64], nor was there a reliable difference in the overall level of recall across the four conditions $[F(3,36)=1.27]$. The reliable interaction supports the observation that the induction procedures differentially influenced the learning of the two word types.

In order to clarify the expected reliable interaction of word type $X$ induction condition, a number of follow-up tests were performed. First, the pattern of recall for the two word types for the participants in the depressed-mood and organizational-schema conditions, was compared. Inspection of the patterns of recall for the depressed-mood and organizational-schema groups in Figure 2 indicates a crossover interaction $\left[F(1,36)=29.53, M S_{\mathrm{e}}=1.10\right]$. Second, the pattern of recall for the two word types for the participants in the arousal and neutral conditions was compared. Inspection of Figure 2 indicates little difference in the pattern of recall for the two words types by the neutral and arousal conditions, which was consistent with our expectations; there was no reliable interaction $[F(1,36)<1.0]$.

The next two tests were performed to demonstrate that the patterns of recall for the depressed and schema groups were reliably different from that of the neutral, control group. The pattern of recall of both the depressed group and the schema group differed reliably from that of the 
neutral group $\left[F(1,36)=5.13, M S_{\mathrm{e}}=1.10\right.$, and $F(1,36)=$ $6.58, M S_{\mathrm{e}}=1.10$, respectively]. The comparisons of patterns of recall of the depressed and schema groups with the neutral group was necessary to demonstrate that not only did the depressed and schema groups show patterns of recall reliably different from one another but also that these patterns of recall for the two word types were reliably different from the pattern of recall displayed by the neutral control group.

Finally, two tests were performed to analyze the level of recall of the two word types in the depressed and schema conditions in order to demonstrate that there was bias to recall one word type over the other in each condition. As can be seen in Figure 2, recall for the negative words was reliably greater than recall for organizational words in the depressed condition $\left[F(1,9)=5.87, M S_{\mathrm{e}}=1.92\right]$. Similarly, recall of the organizational words in the schema condition was reliably greater than that of negative words $[F(1,9)=$ $\left.27.43, M S_{\mathrm{e}}=0.47\right]$. These analyses indicated that the depressed and schema inductions led to a reliable bias to recall one word type over the other.

In summary, we found that the different induction procedures led to differential recall of the two word types. The depressed-mood induction procedure led to the typical mood-congruence effect, producing selective retrieval of negative words over organizational words. The schema induction procedure also led to selective retrieval of the word list, but selection was biased toward the organizational words. Finally, the arousal induction procedure did not lead to selective recall of one word type over the other. Collectively, the findings of Experiment 1 indicate that the cognitive activity associated with the experience of an emotional state mediates the occurrence of mood congruence, whereas physiological arousal has little or no impact on its occurrence.

\section{EXPERIMENT 2}

Experiment 2 was designed to examine further the mediational effect of cognitive activity with respect to mood congruence found in Experiment 1. Experiment 2 focused on the retrieval aspect of mood congruence by examining the influence of the different induction procedures on the recall of previously learned information. This was accomplished by administering the induction procedure after the participants had learned the list of words and by examining the recall of the word list both prior to and after the induction procedure. The expectation was that, prior to the induction procedure, all participants would show the same pattern of recall of the two word types, but, after the induction procedure, the pattern of retrieval for the different conditions would be consistent with that found in Experiment 1 . Finding retrieval patterns as expected would further support the contention that cognitive activity, and not a change in physiological arousal, leads to the selective recall characteristic of mood congruence.

\section{Method}

\section{Participants and Design}

Seventy-two undergraduate students enrolled in introductory psychology classes at the University of New Mexico participated in the experiment. The participants were randomly assigned to one of four conditions that differed with respect to the induction procedure to be utilized: depressed mood, neutral mood, organizational schema, and arousal. Twenty additional participants were excluded from participation in the experiment on the basis of the prescreening procedure

\section{Induction Procedures, Assessment, and Prescreening}

The same induction procedures that were used in Experiment 1 were again used in Experiment 2. The depressed-mood, neutralmood, and schema induction procedures were again presented on an IBM PS/ 2 computer. The arousal induction again consisted of $5 \mathrm{~min}$ of moderate exercise. The DACL (Lubin, 1965) was again used to measure the participant's relative level of depression following the induction procedure, and the participant's HR was measured immediately prior to and following the induction procedure, and, for 10 of the participants in each condition, HR was recorded immediately after the delayed recall task. The BDI (Beck, 1978) was again used to prescreen all potential participants.

\section{Word List}

The same list of words used in Experiment 1 was again used Again, the list was presented twice, in two different random orders

\section{Procedure}

After the participant was seated in front of the computer, he/she was told of the induction procedure to follow and given the option of terminating the experiment at any time. The BDI was then completed by all participants, and their initial $H R$ was taken and noted Following the HR measure, the participants read a series of instructions that described the recall task to follow and were given a sheet of paper on which to recall words. The list of 20 words was presented twice, in two different orders, and then the participants were given up to $3 \mathrm{~min}$ to recall as many words in the list as they could. This recall will be referred to as immediate free recall.

Following the immediate free recall period, the induction procedure corresponding to the participant's assigned condition was administered. The participant's postinduction HR was taken, and the DACL was administered. The participants were then given a blank sheet of paper and instructed to try and recall as many words from the list as they could, subsequently referred to as delayed free recall. They were given up to $3 \mathrm{~min}$ for recall.

Subsequent to the delayed recall period, HR was recorded for 10 of the participants in each condition, and the participants who received a depressed-mood induction were given 10 cards with happy statements on them to read. This latter procedure was done to ensure that no participant left the experiment while still depressed. The participants in the arousal group were allowed to depart after their HR had returned to within $10 \%$ of initial HR.

\section{Results and Discussion}

\section{Comparability of Groups}

The BDI was used to assess the initial comparability across the four groups. The average scores for the depressed, schema, arousal, and neutral groups were 5.2,3.6, 4.7 , and 5.1 , respectively. There was no reliable difference among the groups $(F<1.0)$. On the basis of this screening procedure, the groups can be considered comparable prior to the induction procedures. 
Table 2

\begin{tabular}{|c|c|c|c|c|}
\hline \multirow{3}{*}{$\begin{array}{l}\text { Induction } \\
\text { Condition }\end{array}$} & \multicolumn{4}{|c|}{ Time of Heart Rate Measure } \\
\hline & \multicolumn{2}{|c|}{ Preinduction } & \multicolumn{2}{|c|}{ Postinduction } \\
\hline & $M$ & $S E M$ & $M$ & $S E M$ \\
\hline Depressed & 73.1 & 4.4 & 72.4 & 3.2 \\
\hline Schema & 76.6 & 4.0 & 73.3 & 3.8 \\
\hline Arousal & 73.3 & 3.0 & 104.3 & 6.7 \\
\hline Neutral & 75.5 & 3.3 & 74.5 & 3.0 \\
\hline
\end{tabular}

\section{Mood Assessment}

The mood produced by the different induction procedures was measured by the DACL. The average scores on the DACL for the depressed, schema, arousal, and neutral inductions were $12.6,5.8,5.9$, and 7.6 , respectively. The overall difference among the four groups was reliable $\left[F(3,68)=11.18, M S_{\mathrm{e}}=16.55\right]$. Our expectation was that a reliable mood effect would be due solely to higher scores on the DACL for the participants who participated in the depressed-mood induction; therefore, three planned pairwise comparisons were conducted in order to test this. We compared the score on the DACL for the neutral group with each of the other three groups individually. The comparison of the depressed group's score with the neutral group's score yielded a reliable difference $[F(1,68)=13.90$, $\left.M S_{\mathrm{e}}=16.55\right]$. In contrast, neither the schema group nor the arousal group differed reliably from the control group on the DACL measure $[F(1,68)=1.72$, and $F(1,36)=$ 1.51 , respectively]. In summary, (1) the depressed-mood induction procedure was successful at producing an elevated level of depression, and (2) there was no reliable difference in the measured level of depression among the remaining groups.

\section{Heart Rate Assessment}

HR was a second measure of the effect of the different induction procedures. Table 2 shows the average HR of the four groups prior to and following the induction procedures. Examination of Table 2 reveals that, prior to the induction procedures, there was no reliable difference in HR across groups $(F<1.0)$. Following the inductions, the arousal group showed a greater HR, whereas the remaining groups showed little change. The large increase in $\mathrm{HR}$ for the arousal group from preinduction to postinduction was responsible for a reliable increase in overall $\operatorname{HR}\left[F(1,68)=23.75, M S_{\mathrm{e}}=63.77\right]$. A reliable interaction of time of measure of HR $\times$ induction type $[F(1,68)=$ 37.75, $\left.M S_{\mathrm{e}}=63.77\right]$ and a reliable difference in HR across the four conditions $\left[F(3,68)=7.18, M S_{\mathrm{c}}=63.77\right]$ were also present.

Our expectation was that an increase in HR would occur in the arousal group from preinduction to postinduction and that the remaining three groups would show no such increase in HR; we therefore performed three planned pair- wise comparisons in order to test this. The analyses compared preinduction and postinduction HR for the neutral group with preinduction and postinduction $\mathrm{HR}$ for each of the other three groups. As expected, we found a reliable interaction of induction condition $x$ time of measure when the neutral group was compared with the arousal group $\left[F(1,68)=72.01, M S_{\mathrm{e}}=63.77\right]$. Also, as expected, there was no reliable change in HR over time when the depressed group was compared with the neutral group $(F<1.0)$, nor when the schema group was compared with the neutral group $(F<1.0)$. The major conclusion to draw from these findings is that the arousal induction was successful at producing an aroused physiological state, whereas the remaining conditions produced no change in arousal.

For half of the participants in Experiment 2, HR was measured immediately after the delayed free recall task to determine whether the HR change was maintained through the experiment. The HR for the participants in the arousal condition $(M=85.2)$ remained higher than the HR for the participants in the depressed, schema, and neutral conditions ( $M \mathrm{~s}=74.6,79.6$, and 73.7, respectively). When the participants' HR was compared with their initial HR across the different conditions, there was a time of HR measure $\times$ condition interaction $\left[F(3,36)=17.48, M S_{\mathrm{e}}=\right.$ 13.23], indicating that the HR change from the initial time of measure to the final time of measure was different across the four conditions. In addition to the reliable interaction, HR after the delayed free recall was reliably higher than at the initial time of HR measure $[F(1,36)=$ $5.04, M S_{\mathrm{e}},=13.23$ ]. At the same time, there was no overall difference in HR across the four conditions $(F<1.0)$.

Consistent with the analyses examining the participants' postinduction $\mathrm{HR}$, three follow-up tests were performed comparing the preinduction and final HRs of the experimental conditions to the preinduction and final HRs of the neutral condition. When the arousal condition HRs were compared with the neutral condition HRs, there was a reliable interaction $\left[F(1,36)=27.67, M S_{\mathrm{e}}=13.23\right]$. The remaining two analyses, comparing the depressed and schema conditions with the neutral condition, did not show a reliable interaction $(F<1.0)$. The importance of the HR findings of a reliably higher $\mathrm{HR}$ in the arousal condition, both at the postinduction and final time of measure, is that it demonstrates that the arousal induction procedure was successful at producing and maintaining a change in measured HR.

\section{Analyses of Recall}

Immediate free recall. Immediate free recall took place immediately after the presentation of the word list and prior to the induction procedures. The recall for each of the groups, broken down by word type, can be seen in Table 3. It is apparent that the negative words were recalled reliably better than the organizational words $[F(1,68)=$ $\left.5.36, M S_{\mathrm{e}}=3.18\right]$, indicating that the negative words were 
Table 3

Immediate Recall of the Different Word Types, Negative and Organizational, Broken Down by Induction Type in the Second Experiment

\begin{tabular}{|c|c|c|c|c|}
\hline \multirow[b]{3}{*}{ Induction Condition } & \multicolumn{4}{|c|}{ Word Type } \\
\hline & \multicolumn{2}{|c|}{ Negative } & \multicolumn{2}{|c|}{ Organizational } \\
\hline & $M$ & $S E M$ & $M$ & $S E M$ \\
\hline Depressed & 4.8 & 0.35 & 4.0 & 0.33 \\
\hline Schema & 4,6 & 0.40 & 4.3 & 0.35 \\
\hline Arousal & 4.4 & 0.28 & 3.9 & 0.42 \\
\hline Neutral & 4.8 & 0.38 & 4.6 & 0.28 \\
\hline
\end{tabular}

Note-Immediate recall refers to the initial recall of the word list immediately after study, but prior to administration of the appropriate induction procedure for the condition.

easier to learn. In contrast, there was no reliable difference in recall across the four induction groups $(F<1.0)$, nor was there an interaction of word type $X$ induction type $(F<1.0)$. The lack of a reliable finding with respect to the latter two analyses, in conjunction with the reliable advantage in encoding the negative words, indicates that, although the negative words were being learned to a greater extent than the organizational words, this selective encoding was consistent across all groups.

Delayed free recall. Delayed free recall was a test of retrieval immediately after the induction procedure. The recall for each group broken down by word type can be seen in Figure 3. There appears to be a bias toward recalling negative words over organizational words similar to that seen in immediate free recall, but this bias was not reliable $[F(1,68)=1.49]$. Consistent with immediate free recall, there was no reliable difference in overall recall across the four groups $(F<1.0)$. Importantly, and as expected, there was a reliable interaction of condition $\times$ word type $\left[F(3,68)=2.90, M S_{\mathrm{e}}=1.68\right]$, which is indicative of mood congruence in a list-learning situation. These analyses indicate that the pattern of recall of the two word types was different across the four conditions.

In order to clarify the expected interaction of word type $\times$ condition, a number of follow-up tests were performed. First, the pattern of recall of the two word types for the participants in the depressed and schema conditions was compared. As would be expected in a situation of selective retrieval, the pattern of recall exhibited by the participants in the depressed condition was reliably different from that exhibited by the participants in the schema condition $\left[F(1,68)=8.46, M S_{\mathrm{e}}=1.68\right]$.

The next three comparisolis examined the pattern of recall of the two word types of the depressed, schema, and arousal conditions with that of the neutral condition. The expectation was that the depressed and schema conditions each would have a pattern of recall for the two word types that was different from that of the neutral condition, whereas the arousal condition would not. In con-

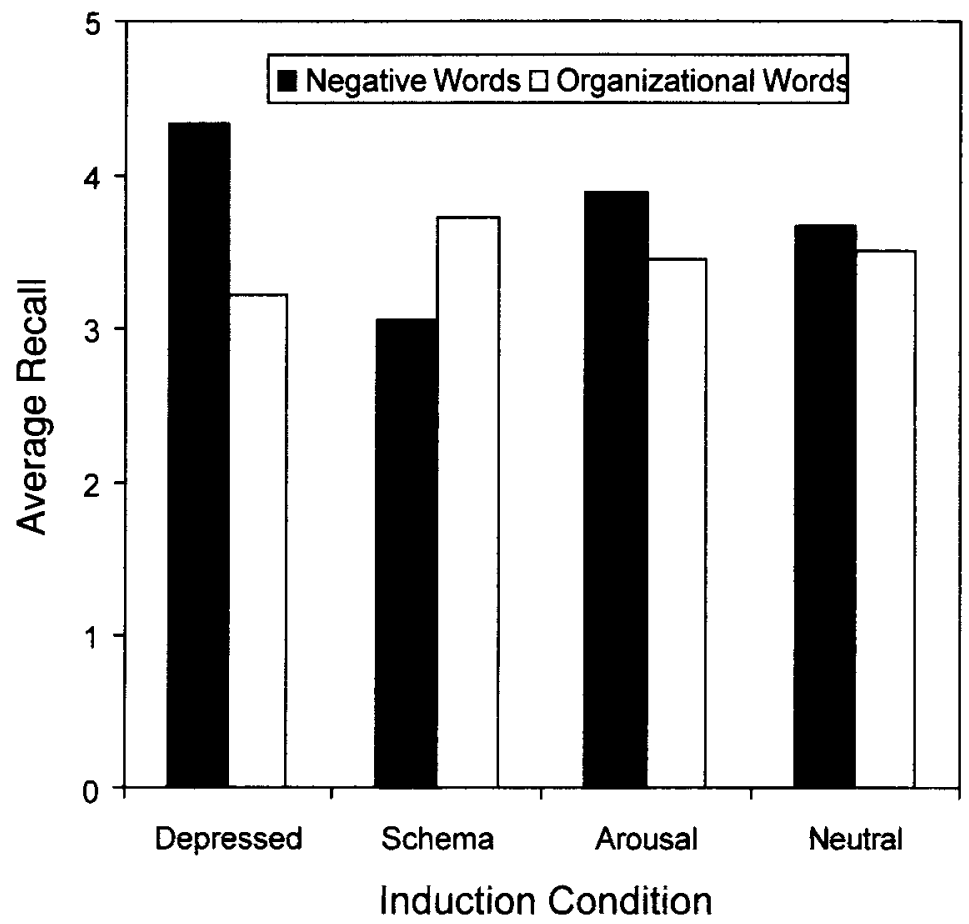

Figure 3. Mean delayed recall for the four induction conditions broken down by word type when examining mood-congruent retrieval. Delayed recall refers to the recall period subsequent to the administration of the appropriate induction procedure. 
trast to expectations, neither the pattern of recall for the depressed condition nor that of the schema condition reliably differed from the pattern of recall of the neutral condition $[F(1,68)=2.39$, and $F(1,68)=1.68$, respectively $]$. The pattern of recall for the arousal condition was also not reliably different from that of the neutral condition $(F<$ 1.0 ), but this was expected.

Finally, two tests were performed to analyze the level of recall of the two word types in the depressed and schema conditions in order to demonstrate that there was bias to recall one word type over the other in each condition. As can be seen in Figure 2, recall for the negative words was reliably greater than recall for organizational words in the depressed condition $\left[F(1,9)=7.26, M S_{\mathrm{e}}=1.38\right]$. In contrast, although there appears to be a small bias to recall organizational words better than negative words in the schema condition, the bias was not reliable $[F(1,9)=2.52$, $\left.M S_{\mathrm{e}}=1.42\right]$. Although the schema condition did not lead to an outright difference in recall of the two word types, the results must be viewed in the context of the overall findings. The finding of a bias to recall negative words over organizational words prior to the induction procedures skewed the delayed recall results toward recalling more negative words than organizational words. Overall, the pattern of selective bias is evident, and an interaction of word type with induction condition was found.

These findings were consistent with expectations: The depressed and schema conditions showed a reliably different pattern of recall of the two word types, which would be indicative of selective retrieval that differed in the two conditions. At the same time, the arousal condition failed to influence retrieval as compared with the neutral condition, indicating that it is unlikely that arousal influences selective retrieval. The patterns of retrieval of the depressed and schema conditions appeared to be different from that of the neutral condition (see Figure 3), but these differences were not great enough to be reliable. The failure to find a reliable difference in pattern of recall for the depressed and schema conditions when compared with the neutral condition, while unexpected, can be explained.

The findings of Experiment 2 parallel the findings of Experiment 1 . Both the depressed group and the schema group exhibited patterns of recall indicative of a retrieval bias, whereas the arousal condition did not. The overall pattern of recall in Experiment 2 was biased toward negative words in the list, but this was consistent across conditions. This bias to encode more of the negative words in the list than organizational words, which was described in the immediate free recall analyses, might have been due to the administration of the BDI immediately prior to the study of the word list. The BDI consists of a series of statements, three fourths of which are negative in content. Reading of a large number of negative statements might have set up a mood-congruent encoding situation for all conditions. Nevertheless, the bias in encoding was con- sistent across all conditions, and the pattern of retrieval at delayed free recall, although biased toward negative words, was supportive of the contention that selective retrieval would occur in the depressed and schema conditions, but not in the arousal condition when compared with the neutral control condition.

\section{GENERAL DISCUSSION}

The findings of the two experiments support the proposal that cognitive activity, as distinct from physiological arousal, mediates the mood congruence phenomenon. Cognitive activity instigated by the administration of a depressed-mood induction procedure led to typical mood congruence results. In addition, and key to supporting our proposal, the administration of the organizationalschema induction procedure led to the selective processing of organizational words over the negative words. Equally important to our thesis, administration of the arousal induction procedure did not lead to selective processing of either word type in the list. The joint finding of cognitive activity leading to selective processing and a change in arousal not leading to selective processing provides strong support for our proposal.

\section{Cognitive Activity Versus \\ Physiological Arousal in Mood Congruence}

The importance of the findings of these experiments is that they distinguish between cognitive and physiological influences on mood congruence. As noted earlier, in order to demonstrate that only one component of an emotional state mediates mood congruence, it was necessary to show that one of the components led to selective processing of information while simultaneously showing that the other had little or no influence on selective processing. Both experiments established that the condition involving cognitive activity alone, the organizational-schema condition, led to selective retrieval of the word list, whereas the condition that solely involved physiological arousal, the arousal condition, showed no selective retrieval of words from the list. These findings were complemented by the typical moodcongruent retrieval pattern found in the depressed-mood condition, which mirrored the retrieval pattern of the organizational-schema condition, and the lack of selective retrieval in the neutral condition, which approximated the pattern of retrieval of the arousal condition. Taken as a whole, the findings indicate that cognitive activity is of central import to the occurrence of mood-congruent processing and that arousal has little or no impact on the selective processing of mood-related information.

\section{Theoretical Implications}

The findings of the two experiments provide a clearer picture of how an emotional state leads to the selective processing of different types of information based on the 
content. Mood congruence can be understood in terms of the cognitive activity that occurs at the time of the processing of information and not one's state of arousal. These findings are consistent with a number of major theories of how an emotional state affects processingspecifically, the resource allocation model (RAM) proposed by Ellis and Ashbrook (1988) and Bower's (1981) semantic network model.

The resource allocation model emphasizes cognitive consequences to account for the effects of an emotional state on memory (Ellis et al., 1993). Cognitive consequences refer to the types of thoughts that occur in conjunction with the experience of an emotion. These thoughts are presumed to be responsible for the effects a mood has on information processing and memory. The manner in which the thoughts impact processing depends on their relationship to the to-be-processed information. The thoughts are inhibitory to processing if they are irrelevant to the information, or they are facilitative if they are relevant (Ellis \& Ashbrook, 1988; Ellis et al., 1993).

The resource allocation model implies that mood congruence will occur only when two conditions are met. First, the material to be processed must contain at least two distinct types of information. This is necessary in order to allow one type of information to be selected at the expense of the other (Blaney, 1986). Second, the thoughts produced must be relevant to the processing of a portion of the information while at the same time being irrelevant to the processing of other information.

Both of these requirements were met in two of the conditions, depressed mood and organizational schema, and not in the other two, arousal and neutral. The depressedmood and organization-schema conditions each involved having the participants focus on a particular idea. In the depressed condition, the participants focused on a negative emotional state that was relevant to the negative words and irrelevant to the organizational words, whereas the participants that focused on writing a paper were focusing on thoughts relevant to the organizational words and irrelevant to the negative words. In both cases, recall of words relevant to the focus of the participants' thoughts were recalled better than the words that were irrelevant. In contrast, the cognitive focus of the participants in the arousal and neutral conditions was most likely idiosyncratic and unrelated to a particular portion of the list. The lack of a recall bias reflected the lack of a relationship oi the words to the thoughts they experienced.

A second theoretical framework that can account for the findings is Bower's (1981) semantic network theory. His theory assumes that emotions are represented as nodes in a network and that each emotion node is linked with propositions that describe events associated with the emotion. Emotion nodes can be activated by a variety of events, and they are also subject to the spread of activation. Network theory has been useful in interpreting mood congruence findings in a number of settings (see
Blaney, 1986). Mood congruence is seen as the activation of emotion nodes by some emotional event that is consistent with the individual's established network, and the activation of these nodes facilitates the retrieval of mood-related information. Network theory can also explain the selective recall findings associated with the organizational-schema condition in a similar fashion. The organizational-schema condition would lead to the activation of nodes in the network that represent the information associated with writing a paper. The activated information representing writing a paper is consistent with the organizational words in the list, and it facilitates the retrieval of those words. The neutral condition, on the other hand, is designed so as not to produce organized cognitions related to a particular concept. This results in random or idiosyncratic activation of the participant's semantic network, and it most likely does not facilitate the retrieval of words in the list. The arousal condition provides the biggest challenge to network theory. If arousal is represented by a node in a semantic network, then it should lead to the activation of associated arousal related information. This activation should lead to the facilitated retrieval of arousal related words. Since emotions involve both cognitive and physiological aspects, then the study and recall of the negative words, being emotional in content, should involve some physiological reaction. Therefore, arousal should facilitate to the retrieval of negative words. There are two possible explanations within network theory that can account for why arousal did not facilitate the retrieval of the negative words. First, the arousal component of the negative words might not have been stored as part of the participants' semantic network. Second, the activation of the negative words and the activation spreading from the arousal node due to the exercise might not have overlapped, precluding facilitation. The latter is more likely since there was a limited time allowed to study the words, and most of the negative words probably did not have a very intense level of arousal associated with them. Overall, network theory accounts for selective recall in the general sense and also predicts when arousal should lead to selective recall.

\section{Conclusions}

In summary, these experiments provide important information about the mechanisms producing mood congruence in memory. Both experiments demonstrated that thoughts an individual has while experiencing an emotional state are of central import to the selective retrieval representative of mood congruence. In addition, the findings indicate that physiological arousal has little or no influence on the selective recall of emotionally toned words that is indicative of mood congruence. $\mathrm{Al}$ though the findings do not favor one theoretical account of emotion and memory over the others, they do provide critical information about how emotional states influence memory. 


\section{REFERENCES}

BECK, A. T. (1978). Depression inventory. Philadelphia: Center for Cognitive Therapy.

Blaney, P. H. (1986). Affect and memory: A review. Psychological Bulletin, 99, 229-248.

Bower, G. H. (1981). Mood and Memory. American Psychologist, 36, 129-149.

Bower, G. H., Gilligan, S. G., \& Monteiro, K. P. (1981). Selectivity in learning caused by affective states. Journal of Experimental Psychology: General, 110, 451-473.

BOWER, G. H., \& MAYER, J. D. (1989). In search of mood-dependent retrieval [Special issue]. Journal of Social Behavior \& Personality, 4, 121-156.

Clark, D. M., \& Teasdale, J. D. (1982). Diurnal variation in clinical depression and accessibility of memories of positive and negative experiences. Journal of Abnormal Psychology, 91, 87-95.

Clark, M. S., Milberg, S., \& Erber, R. (1988). Arousal-state-dependent memory: Evidence and implications for understanding social judgments and social behavior. In K. Fiedler \& J. Forgas (Eds.), Affect, cognition, and social behavior (pp. 63-83). Toronto: Hogrefe.

Clark, M. S., Milberg, S., \& Ross, J. (1983). Arousal cues arousalrelated material in memory: Implications for understanding effects of mood on memory. Journal of Verbal Learning \& Verbal Behavior, 22, 633-649.

Ellis, H. C., \& AshBrooK, P. W. (1988). Resource allocation model of the effects of depressed mood states on memory. In K. Fiedler \& J. Forgas (Eds.), Affect, cognition, and social behavior (pp. 25-43). Toronto: Hogrefe.

Ellis, H. C., Seibert, P. S., \& VARner, L. J. (1995). Emotion and memory: Effects of mood states on immediated and unexpected delayed recall. Journal of Social Behavior \& Personality, 10, 349-362.

Ellis, H. C., Varner, L. J., \& BeCker, A. S. (1993). Cognition and emotion: Theories, implications, and educational applications. In L. A. Penner, G. M. Batsche, H. M. Knoff, \& D. L. Nelson (Eds.), The challenge in mathematics and science education: Psychology's response (pp. 83-111). Washington, DC: American Psychological Association.

Ellis, H. C., Varner, L. J., Becker, A. S., \& Ottaway, S. A. (1995). Emotion and prior knowledge in memory and judged comprehension of ambiguous stories. Cognition \& Emotion, 9, 363-382.

Fiedler, K., \& Stroehm, W. (1986). What kind of mood influences what kind of memory: The role of arousal and information structure. Memory \& Cognition, 14, 181-188.

Gilligan, S. G., \& Bower, G. H. (1983). Reminding and moodcongruent memory. Bulletin of the Psychonomic Society, 21, 431-434.

LuBin, B. (1965). Adjective checklists for measurement of depression. Archives of General Psychiatry, 12, 57-62.

MANDLER, G. (1992). Memory, arousal, and mood: A theoretical integration. In S. A. Christianson (Ed.), The handbook of emotion and memory: Research and theory (pp. 93-110). Hillsdale, NJ: Erlbaum.

MARTIN, M., HORDER, P., \& JoNES, G. V. (1992). Integral bias in naming phobia-related words. Cognition \& Emotion, 6, 479-486.

Mayer, J. D., \& SaloveY, P. (1988). Personality moderates the interaction of mood and cognition. In K. Fiedler \& J. Forgas (Eds.), Affect, cognition. and social behavior (pp. 87-99). Toronto: Hogrefe.

Mayer, J. D., \& Volanth, A. J. (1985). Cognitive involvement in the mood response system. Motivation \& Emotion, 9, 261-275.

Paivio, A., Yuille, J. C., \& Madigan, S. A. (1968). Concreteness, imagery, and meaningfulness values of 925 nouns. Journal of Experimental Psychology Monographs, 76 (1, Pt. 2).

Parrott, W. G. (1991). Mood induction and instructions to sustain moods: A test of the subject compliance hypothesis of mood-congruent memory. Cognition \& Emotion, 5, 4|-52.

Perrig, W. J., \& Perrig, P. (1988). Mood and memory: Mood-congruity effects in absence of mood. Memory \& Cognition, 16, 102-109.

Revelle, W. \& Loftus, D. A. (1990). Individual differences and arousal: Implications for the study of mood and memory. Cognition \& Emotion, 4, 209-237.

Revelle, W., \& Loftus, D. A. (1992). The implications of arousal effects for the study of affect and memory. In S. Christianson (Ed.), The handbook of emotion and memory: Research and theory (pp. 113-149). Hillsdale, NJ: Erlbaum.

RISKIND, W. H. (1989). The mediating mechanisms in mood and memory: A cognitive-priming formulation. Journal of Social Behavior \& Personality, 4, 173-184.

ROTHKOPF, J. S., \& BLANEY, P. H. (1991). Mood-congruent memory: The role of affective focus and gender. Cognition \& Emotion, 5, 53-64.

SAlovey, P., \& Singer, J. A. (1989). Mood congruency effects in recall of childhood versus recent memories. Journal of Social Behavior \& Personality, 4, 99-120.

SChaCter, S., \& Singer, J. E. (1962). Cognitive, social, and physiological determinants of emotional state. Psychological Review, 69, 379-399.

SeiberT, P. S., \& ElLIS, H. C. (1991). A convenient self-referencing mood induction procedure. Bulletin of the Psychonomic Society, 29, 121-124.

ZaJONC, R. B. (1980). Feeling and thinking: Preferences need no inferences. American Psychologist, 35, 151-175.

\section{APPENDIX A}

\section{Instructions for Schema Induction Condition}

I will read each of the following cards to myself and then I will read the card aloud. In this part of the experiment I will be reading a series of cards with statements typed on them. These statements represent a certain idea or action. In order to participate fully and successfully, I will need to be willing to experience each statement as it would apply to me personally. In other words, when I read each statement, I will allow myself to respond as though the statement had been my own original thought. I will think about each idea without interference-I will relate to the idea presented.

At first I might feel like resisting this procedure of relating to the idea presented, many people feel this way. However, I will see that it is the case that I may have the opportunity to learn something about the idea. Thus I will try to relate to the idea presented.

I will relate to the idea, making the statement my own with as much realism as possible. I will move into the frame of mind required to relate to the idea and will not attempt to stop it. I will visualize a scene when I may have participated in an action associated with the idea. Then I will say whatever comes to my mind that relates to the idea. This is a type of free association, letting thoughts that pertain to the idea flow freely.

I am now ready to relate to the statements that follow. From this point forward, whenever I hear the tone, I will go on to the next card. I will spend the time between the tones reading the statements and relating to the ideas they suggest to me. I am ready to begin.

\section{Schema Induction Statements}

1. I find that writing a paper can be very complicated.

2. I often write several drafts of my papers.

3. I learn something from every paper I write.

4. The first sentence is always the hardest for me to write.

5. Writing a paper is fun if $I$ like the topic.

6. I must eventually proof read my paper carefully.

7. I find it difficult to organize my ideas.

8. I must decide on my topic.

9. I write a little bit at a time. 
10. I don't like to be required to write papers.

11. I write better when I am in a good mood.

12. Making revisions always discourages me.

13. Researching my idea is very important for success

14. The more I write the better I get.

15. Essays are the fairest ways to test my knowledge.

16. I can express my opinions and feelings when I write.

17. Many of my classes require me to write a paper.

18. I must take time to express my ideas clearly.

19. It can take a lot of time for me to write a paper.

20. I sometimes have problems with grammar, but I get better with practice.

21. The length of my papers depend on the topic.

22. It is sometimes difficult to find the correct words to use.

23. Writing can be very stressful for me.

24. My typewriter and I are not always friends.

25. Writing is one of my creative outlets.

\begin{tabular}{cc}
\multicolumn{2}{c}{$\begin{array}{c}\text { APPENDIX B } \\
\text { Word List }\end{array}$} \\
\hline Organizational Words & Negative Words \\
\hline CONCEPT & DECEIT \\
SATIRE & BETRAYAL \\
THEORY & SHAME \\
KNOWLEDGE & DEATH \\
METHOD & TRAGEDY \\
IDEA & FATE \\
EFFORT & GRIEF \\
MORAL & ANXIETY \\
OPINION & SADNESS \\
MIND & WISTFUL \\
\hline
\end{tabular}

(Manuscript received February 21, 1996; revision accepted for publication April 20, 1997.) 